\title{
A Fast Algorithm for Reconstructing $h v$-Convex 8-Connected but Not 4-Connected Discrete Sets
}

\author{
Péter Balázs, Emese Balogh, and Attila Kuba \\ Department of Informatics, University of Szeged \\ Árpád tér 2, H-6720 Szeged, Hungary \\ \{pbalazs, bmse, kuba\}@inf .u-szeged.hu
}

\begin{abstract}
One important class of discrete sets where the reconstruction from two given projections can be solved in polynomial time is the class of $h v$-convex 8 -connected sets. The worst case complexity of the fastest algorithm known so far for solving the problem is of $O(m n$. $\min \left\{m^{2}, n^{2}\right\}$ ) 2. However, as it is shown, in the case of 8-connected but not 4-connected sets we can give an algorithm with worst case complexity of $O(m n \cdot \min \{m, n\})$ by identifying the so-called $\mathcal{S}_{4}$-components of the discrete set. Experimental results are also presented in order to investigate the average execution time of our algorithm.
\end{abstract}

Keywords: Discrete tomography, reconstruction, convex and connected discrete set.

\section{Introduction}

One of the most frequently studied area of discrete tomography [8]9] is the problems of the reconstruction of 2-dimensional (2D) discrete sets from their row and column sum vectors. There are reconstruction algorithms for different classes of discrete sets (e.g., 34671011,14]). However, the reconstruction in certain classes can be NP-hard (see [15]). Since applications require fast algorithms, it is important to find reconstruction algorithms in those classes of $2 \mathrm{D}$ discrete sets where the reconstruction can be performed in polynomial time.

We always suppose having some a priori information of the set to be reconstructed. The most frequently used properties are connectedness, directedness and some kind of discrete versions of the convexity.

One important class where the reconstruction problem from two given projections can be solved in polynomial time is the class of $h v$-convex 8-connected sets. Several algorithms have been developed for solving this problem [511, among them the fastest has worst case complexity of $O\left(m n \cdot \min \left\{m^{2}, n^{2}\right\}\right)[2]$. In this paper we give an algorithm with worst case complexity of $O(m n \cdot \min \{m, n\})$ for the reconstruction problem in the class of $h v$-convex 8-connected but not 4-connected sets by examining the features of these sets.

This paper is structured as follows. First, the necessary definitions are introduced in Section 2 In Subsection 3.1 we define $\mathcal{S}_{4}$-components of an 8connected but not 4 -connected $h v$-convex set and prove some properties of them, 
then, in Subsection 3.2 we investigate the directedness of these components. $\mathcal{S}_{4^{-}}$ components can be identified from two given projections, as it is shown in Subsection 3.3. The reconstruction algorithm is represented in Subsection 3.4, the experimental results are given in Subsection 3.5 .

\section{Definitions and Notation}

The finite subsets of $\mathbb{Z}^{2}$ (the $2 \mathrm{D}$ integer lattice) are called discrete sets, its elements are called points or positions. $\mathcal{F}$ denotes the class of discrete sets.

In the followings discrete sets will be represented by binary matrices $F=$ $\left(f_{i j}\right)_{m \times n}$, where $f_{i j} \in\{0,1\}$. Figure [1] shows a discrete set represented by the binary matrix

$$
F=\left(\begin{array}{llllll}
0 & 1 & 0 & 0 & 0 & 0 \\
0 & 1 & 0 & 0 & 0 & 0 \\
1 & 1 & 0 & 0 & 0 & 0 \\
0 & 1 & 1 & 0 & 0 & 0 \\
0 & 0 & 0 & 1 & 1 & 1
\end{array}\right)
$$

For any discrete set $F$ we define its projections by the operations $\mathcal{H}$ and $\mathcal{V}$ as follows. $\mathcal{H}: \mathcal{F} \longrightarrow \mathbb{N}_{0}^{m}, \mathcal{H}(F)=H=\left(h_{1}, \ldots, h_{m}\right)$, where

$$
h_{i}=\sum_{j=1}^{n} f_{i j}, \quad i=1, \ldots, m,
$$

and $\mathcal{V}: \mathcal{F} \longrightarrow \mathbb{N}_{0}^{n}, \mathcal{V}(F)=V=\left(v_{1}, \ldots, v_{n}\right)$, where

$$
v_{j}=\sum_{i=1}^{m} f_{i j}, \quad j=1, \ldots, n .
$$

The vectors $H$ and $V$ are called the row and column sum vectors of $F$, respectively (see Fig. 1). $H$ and $V$ are also called the projections of $F$.

Not any pair of vectors is the projections of some discrete set. In the followings we suppose, that $H \in \mathbb{N}_{0}^{m}$ and $V \in \mathbb{N}_{0}^{n}$ are compatible which means that they satisfy the following two conditions.

(i) $h_{i} \leq n$, for $1 \leq i \leq m$, and $v_{j} \leq m$, for $1 \leq j \leq n$;

(ii) $\sum_{i=1}^{m} h_{i}=\sum_{j=1}^{n} \bar{v}_{j}$, i.e., the two vectors have the same total sums.

The cumulated vectors of $H$ and $V$ are denoted by $\widetilde{H}=\left(\widetilde{h}_{1}, \ldots, \widetilde{h}_{m}\right)$ and $\widetilde{V}=\left(\widetilde{v}_{1}, \ldots, \widetilde{v}_{n}\right)$, respectively, and defined with the following recursive formulas,

$$
\begin{array}{cc}
\widetilde{h}_{1}=h_{1}, & \widetilde{h}_{i}=\widetilde{h}_{i-1}+h_{i}, \quad i=2, \ldots, m, \\
\widetilde{v}_{1}=v_{1}, \quad & \widetilde{v}_{j}=\widetilde{v}_{j-1}+v_{j}, \quad j=2, \ldots, n
\end{array}
$$

(see Fig. 1).

Given a class $\mathcal{G}$ of discrete sets, we say that the discrete set $F \in \mathcal{G}$ is unique in the class $\mathcal{G}$ (w.r.t. the row and column sum vectors) if there is no different discrete set $F^{\prime} \in \mathcal{G}$ for which $\mathcal{H}(F)=\mathcal{H}\left(F^{\prime}\right)$ and $\mathcal{V}(F)=\mathcal{V}\left(F^{\prime}\right)$. 


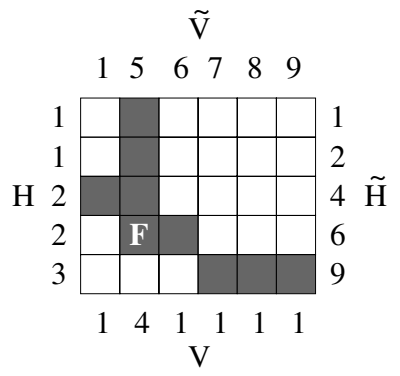

Fig. 1. An $h v$-convex 8- but not 4-connected discrete set $F$. The projections of $F$ are the vectors $H$ and $V$, the cumulated vectors of $H$ and $V$ are denoted by $\widetilde{H}$ and $\widetilde{V}$.

Two points $P=\left(p_{1}, p_{2}\right)$ and $Q=\left(q_{1}, q_{2}\right)$ in $\mathbb{Z}^{2}$ are said to be 4-adjacent if $\left|p_{1}-q_{1}\right|+\left|p_{2}-q_{2}\right|=1$. The points $P$ and $Q$ are said to be 8 -adjacent if they are 4-adjacent or $\left|p_{1}-q_{1}\right|=1$ and $\left|p_{2}-q_{2}\right|=1$. The sequence of distinct points $\left(i^{(0)}, j^{(0)}\right), \ldots,\left(i^{(k)}, j^{(k)}\right)$ is a $4 / 8$-path from point $\left(i^{(0)}, j^{(0)}\right)$ to point $\left(i^{(k)}, j^{(k)}\right)$ in a discrete set $F$ if each point of the sequence is in $F$ and $\left(i^{(l)}, j^{(l)}\right)$ is $4 / 8$ adjacent, respectively, to $\left(i^{(l-1)}, j^{(l-1)}\right)$ for each $l=1, \ldots, k$. Two points are 4/8-connected in the discrete set $F$ if there is a 4/8-path, respectively, in $F$ between them. A discrete set $F$ is $4 / 8$-connected if any two points in $F$ are 4/8-connected, respectively, in $F$. The 4-connected set is also called polyomino. The discrete set $F$ is $h$-convex/v-convex if its rows/columns are 4 -connected, respectively. The $h$ - and $v$-convex sets are called $h v$-convex (see Fig. 11).

We denote the class of $h v$-convex 8-connected and $h v$-convex 4-connected discrete sets by $\mathcal{S}_{8}$ and $\mathcal{S}_{4}$, respectively. Clearly, $\mathcal{S}_{8} \supset \mathcal{S}_{4}$ (see, e.g., Fig. 团) and so $\mathcal{S}_{8} \backslash \mathcal{S}_{4} \neq \emptyset$. Let $\mathcal{S}_{8}^{\prime}=\mathcal{S}_{8} \backslash \mathcal{S}_{4}$. In this paper we are going to study the problem of reconstruction in the class of $h v$-convex 8 - but not 4 -connected discrete sets $\operatorname{Reconstruction}\left(\mathcal{S}_{8}^{\prime}\right)$.

Instance: $\quad$ Two compatible vectors $H \in \mathbb{N}_{0}^{m}$ and $V \in \mathbb{N}_{0}^{n}$.

Task: $\quad$ Construct a discrete set $F \in \mathcal{S}_{8}^{\prime}$ such that $\mathcal{H}(F)=H$ and $\mathcal{V}(F)=V$.

Note, that components of the row and column sum vectors of an 8-connected set cannot be zero, therefore in the followings we assume that the input of the above problem are the vectors $H \in \mathbb{N}^{m}$ and $V \in \mathbb{N}^{n}$.

\section{Reconstruction of Sets of $\mathcal{S}_{8}^{\prime}$}

\section{$3.1 \quad \mathcal{S}_{4}$-Components}

Let $F \in \mathcal{S}_{8}^{\prime}$. A maximal $h v$-convex 4 -connected subset of $F$ is called an $\mathcal{S}_{4^{-}}$ component of $F$. Clearly, the $\mathcal{S}_{4}$-components $F_{1}, \ldots, F_{k}$ of $F$ give a uniquely determined partition of $F$ and the number of $\mathcal{S}_{4}$-components of $F$ is at least 2 (see, e.g., Fig. 1 where there are two $\mathcal{S}_{4}$-components: $\{(5,4),(5,5),(5,6)\}$ and $\{(1,2),(2,2),(3,1),(3,2),(4,2),(4,3)\})$. 
Since $F$ is $h v$-convex, the sets of the row/column indices of the elements of $F_{1}, \ldots, F_{k}$ consist of consecutive integers and they are disjoint. Then it follows that there is an $\mathcal{S}_{4}$-component of $F$, say $F_{1}$ with the smallest containing discrete rectangle $(\mathrm{SCDR}) R_{1}=I_{1} \times J_{1}$ such that $I_{1}=\left\{1, \ldots, i_{1}\right\}$ for some $i_{1} \geq 1$. Similarily, we get that there is another $\mathcal{S}_{4}$-component of $F$, say $F_{2}$ with the $\operatorname{SCDR} R_{2}=I_{2} \times J_{2}$ such that $I_{2}=\left\{i_{1}+1, \ldots, i_{2}\right\}$ for some $i_{2}>i_{1}$. And so on. Generally, there are integers $0=i_{0}<i_{1}<\ldots<i_{k-1}<i_{k}=m(k \geq 2)$ such that $I_{l}=\left\{i_{l-1}+1, \ldots, i_{l}\right\}$ contains the row indices of the $l$-th $\mathcal{S}_{4}$-component of $F$ for each $l(1 \leq l \leq k)$. Among $I_{1}, \ldots, I_{k}$ we define a relation " $<$ " as follows. Let $I, I^{\prime} \in\left\{I_{1}, \ldots, I_{k}\right\}$. We say that $I<I^{\prime}$ if each element of $I$ is less than any element of $I^{\prime}$. Using this relation we can write shortly that

$$
I_{1}<I_{2}<\ldots<I_{k} .
$$

We define the same relation among $J_{1}, \ldots, J_{k}$. In order to give a description of the relative positions of the $\mathcal{S}_{4}$-components of $F$ consider

Theorem 1. Let $F \in \mathcal{S}_{8}^{\prime}$ having $\mathcal{S}_{4}$-components $F_{1}, \ldots, F_{k}$ with the SCDRs $I_{1} \times J_{1}, \ldots, I_{k} \times J_{k}(k \geq 2)$ such that (5) is satisfied. Then exactly one of the following cases is possible.

Case 1. $J_{1}<J_{2}<\ldots<J_{k}$,

Case 2. $J_{1}>J_{2}>\ldots>J_{k}$.

Proof. The proof is quite technical (see [1]).

In the followings we say that $F \in \mathcal{S}_{8}^{\prime}$ has type 1 if Case 1 of Theorem 1 is satisfied, otherwise, that is, if Case 2 of Theorem 1 is satisfied, it has type 2 . As an example see Fig. 2.

$\mathrm{F}$

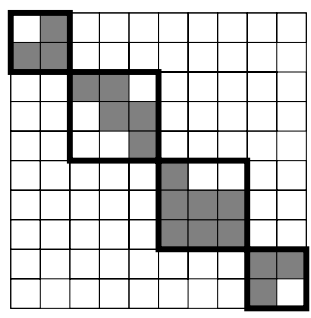

$F^{\prime}$

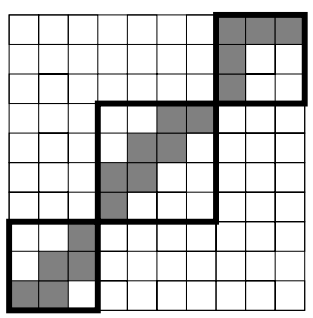

Fig. 2. A discrete set $F$ of type 1 and a discrete set $F^{\prime}$ of type 2. The SCDRs are drawn with bold lines. $C_{F}=\{(2,2),(5,5),(8,8)\}, C_{F^{\prime}}=\{(3,8),(7,4)\}$.

Corollary 1. Let $F \in \mathcal{S}_{8}^{\prime}$. Then there are uniquely determined row indices $0=$ $i_{0}<i_{1}<\ldots<i_{k}=m$ and column indices $0=j_{0}<j_{1}<\ldots<j_{k}=n$ such that $I_{l} \times J_{l}$ is the $S C D R$ of the $\mathcal{S}_{4}$-component $F_{l}$ of $F$ for each $l=1, \ldots, k(k \geq 2)$, where $I_{l}=\left\{i_{l-1}+1, \ldots, i_{l}\right\}$ and

$$
J_{l}= \begin{cases}\left\{j_{l-1}+1, \ldots, j_{l}\right\}, & \text { if } F \text { has type } 1, \\ \left\{j_{k-l}+1, \ldots, j_{k-l+1}\right\}, & \text { if } F \text { has type } 2 .\end{cases}
$$




\subsection{Directed Discrete Sets}

An 8-path in a discrete set $F$ is an NE-path from point $\left(i^{(0)}, j^{(0)}\right)$ to point $\left(i^{(t)}, j^{(t)}\right)$ if each point $\left(i^{(l)}, j^{(l)}\right)$ of the path is in north or east or northeast to $\left(i^{(l-1)}, j^{(l-1)}\right)$ for each $l=1, \ldots, t$. $S W$-, $S E$-, $N W$-paths can be defined similarily. The discrete set $F$ is $N E$-directed if there is a particular point of $F$, called source (which is necessarily the point $(m, 1)$ ), such that there is an $N E$ path in $F$ from the source to any other point of $F$. Similar definitions can be given for $S W$-, $S E$ - and $N W$-directedness. The discrete set $F$ in Fig. 1 is $N W$ directed with source $(5,6)$. On the base of the following lemma, it is easy to check the directedness of discrete sets in the class $\mathcal{S}_{4}$.

Lemma 1. Let $G \in \mathcal{S}_{4}$ and $R=\left\{i^{\prime}+1, \ldots, i^{\prime \prime}\right\} \times\left\{j^{\prime}+1, \ldots, j^{\prime \prime}\right\}\left(i^{\prime}<i^{\prime \prime}, j^{\prime}<j^{\prime \prime}\right)$ be its $S C D R$.

(i) $G$ is $S E$-directed if and only if $g_{i^{\prime}+1, j^{\prime}+1}=1$;

(ii) $G$ is $N W$-directed if and only if $g_{i^{\prime \prime}, j^{\prime \prime}}=1$;

(iii) $G$ is $S W$-directed if and only if $g_{i^{\prime}+1, j^{\prime \prime}}=1$;

(iv) $G$ is $N E$-directed if and only if $g_{i^{\prime \prime}, j^{\prime}+1}=1$.

Proof. It follows from the definitions directly. (As an example see Fig. 2).

Theorem 2. Let $F \in \mathcal{S}_{8}^{\prime}$ having $\mathcal{S}_{4}$-components $F_{1}, \ldots, F_{k}(k \geq 2)$. If $F$ has type 1 then $F_{1}, \ldots, F_{k-1}$ are $N W$-directed and $F_{2}, \ldots, F_{k}$ are $S E$-directed. If $F$ has type 2 then $F_{1}, \ldots, F_{k-1}$ are $N E$-directed and $F_{2}, \ldots, F_{k}$ are $S W$-directed.

Proof. The proof is based on Corollary 1 and Lemma 1] (see [1]).

Depending on the type of $F$ let us define

$$
C_{F}= \begin{cases}\left\{\left(i_{l}, j_{l}\right) \mid l=1, \ldots, k-1\right\}, & \text { if } F \text { has type } 1, \\ \left\{\left(i_{l}, j_{k-l}+1\right) \mid l=1, \ldots, k-1\right\}, & \text { if } F \text { has type } 2\end{cases}
$$

where $i_{1}, \ldots, i_{k-1}$ and $j_{1}, \ldots, j_{k-1}$ denote the uniquely determined indices mentioned in Corollary 1 That is, $C_{F}$ consists of the sources of the $N W-/ N E$ directed $\mathcal{S}_{4}$-components $F_{1}, \ldots, F_{k-1}$ if $F$ has type 1/2, respectively (see Fig. (2).

The knowledge of any element of $C_{F}$ is useful in the reconstruction of an $F \in$ $\mathcal{S}_{8}^{\prime}$, as we can see on the base of the following theorem.

Theorem 3. Any $F \in \mathcal{S}_{8}^{\prime}$ is uniquely determined by its projections, its type and an arbitrary element of $C_{F}$.

Proof. The proof is based on Theorem 3 in [12] and Theorem 2 (see [1]).

Corollary 2. If $F, F^{\prime} \in \mathcal{S}_{8}^{\prime}$ are different solutions of the same reconstruction problem and they have the same type then $C_{F} \cap C_{F^{\prime}}=\emptyset$. 


\subsection{Equality Positions}

Let $\widetilde{H}$ and $\widetilde{V}$ be the cumulated vectors of the projections of $F \in \mathcal{S}_{8}^{\prime}$. We say that $(i, j) \in\{1, \ldots, m\} \times\{1, \ldots, n\}$ is an equality position of type 1 if $\widetilde{h}_{i}=\widetilde{v}_{j}$. $(m, n)$ is a trivial equality position of type 1 and in the followings we omit it. We say that $(i, j) \in\{1, \ldots, m\} \times\{2, \ldots, n+1\}$ is an equality position of type 2 if $\widetilde{h}_{i}=\widetilde{v}_{n}-\widetilde{v}_{j-1}$. Not every equality position is in $C_{F}$ but they are useful to find the elements of $C_{F}$ (see Fig. 31).

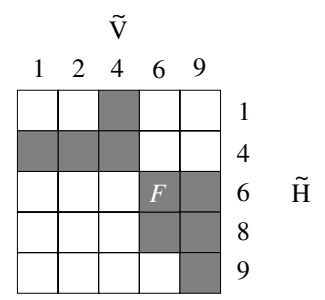

Fig. 3. A discrete set $F$ with cumulated vectors $\widetilde{H}$ and $\widetilde{V}$. $(1,1),(2,3)$ and $(3,4)$ are equality positions of type 1 . However, only $(2,3)$ is in $C_{F}$. $(4,2)$ is the only equality position of type 2 but it is not in $C_{F}$ since $F$ has type 1 .

Lemma 2. Let $F \in \mathcal{S}_{8}^{\prime}$ and $C_{F}$ be defined by (9). Then the elements of $C_{F}$ are all equality positions of the same type as of $F$.

Proof. See [1].

\subsection{The Reconstruction Algorithm}

Our algorithm is called Algorithm REC8' and works as follows. We first assume that the set $F \in \mathcal{S}_{8}^{\prime}$ to be reconstructed has type 1. On the base of Theorem 3 it is sufficient to find an arbitrary element of $C_{F}$ to reconstruct $F$ from its projections uniquely. The elements of $C_{F}$ are equality positions of type 1 on the base of Lemma 2. So, in order to find all solutions of the reconstruction problem, we start to check every equality position of type 1 whether it is an element of $C_{F}$ and if it is then we find a solution. The set $L_{1}$ of equality positions of type 1 can be found by the comparison of the cumulated row and column sums. This algorithm is called Algorithm L_1 and it is similar to the procedure used for reconstructing the spine of $h v$-convex polyominoes [12. An analogous algorithm can be given to find all equality positions of type 2 (Algorithm L_2).

Since the knowledge of any element of $C_{F}$ is sufficient, again on the base of Theorem 3, without losing any solution, we can assume that if an investigated equality position $(i, j)$ of type 1 is in $C_{F}$ then it is the source of the first $\mathcal{S}_{4}$-component $F_{1}$, i.e., the one with the $\operatorname{SCDR}\left\{1, \ldots, i_{1}\right\} \times\left\{1, \ldots, j_{1}\right\}$, i.e., $(i, j)=\left(i_{1}, j_{1}\right)$. On the base of Theorem 2 this $\mathcal{S}_{4}$-component is $N W$-directed. Now, in order to decide if $(i, j)$ is the source of $F_{1}$ we try to reconstruct an $h v$ convex $N W$-directed polyomino with source $(i, j)$. This can be done using Algorithm RecNW which is a simple modification of the algorithm for reconstructing 
directed discrete sets given in [12. Algorithm RecNW tries to reconstruct an $m \times n$ binary matrix $G$ from the input data $H, V$ and $(i, j)$ such that the 1 's of $G$ constitute an $h v$-convex $N W$-directed polyomino having source $(i, j)$ and the row and column sums of $G$ in the non-zero rows and columns are equal to the corresponding elements of $H$ and $V$, respectively. If RecNW can reconstruct such a $G$ then it returns also the upper left position $\left(i^{\prime}, j^{\prime}\right)$ of the SCDR of $G$. If RecNW fails, there is no such a binary matrix $G$. Now, there are two cases:

(1) RecNW fails. Clearly, in this case $(i, j)$ cannot be the source of $F_{1}$. We continue with the investigation of the next equality position from $L_{1}$.

(2) RecNW gives a (unique) solution, i.e., it is possible to reconstruct an $h v$ convex $N W$-directed polyomino $G$ with source $(i, j)$ and with the SCDR $\left\{i^{\prime}, \ldots, i\right\} \times\left\{j^{\prime}, \ldots, j\right\}$, where $1 \leq i^{\prime} \leq i=i_{1}$ and $1 \leq j^{\prime} \leq j=j_{1}$. If $\left(i^{\prime}, j^{\prime}\right) \neq$ $(1,1)$ then, clearly $G$ cannot be the first $\mathcal{S}_{4}$-component of $F$, i.e., $F_{1} \neq G$ and we continue with the investigation of the next equality position from $L_{1}$. Otherwise, i.e., when $\left(i^{\prime}, j^{\prime}\right)=(1,1)$, we can assume that $F_{1}=G$ and we try to reconstruct the $2 \mathrm{nd}, 3 \mathrm{rd}, \ldots \mathcal{S}_{4}$-components iteratively. Reconstruction of the $S E$-directed $k$-th component $F_{k}(k=2, \ldots)$ can be done using Algorithm RecSE. Algorithm RecSE tries to reconstruct an $m \times n$ binary matrix $G$ from the input data $H, V$ and $(i, j)$ such that the 1 's of $G$ constitute an $h v$-convex $S E$-directed polyomino having source $(i, j)$ and the row and column sums of $G$ in the non-zero rows and columns are equal to the corresponding elements of $H$ and $V$, respectively. If RecSE can reconstruct such a $G$ then it returns also the lower right position $\left(i^{\prime}, j^{\prime}\right)$ of the SCDR of $G$. If RecSE fails, there is no such binary matrix $G$. On the base of Theorem 2, $F_{k}$ must be $S E$ directed with source $\left(i_{k-1}+1, j_{k-1}+1\right)$. We call RecSE to reconstruct such a polyomino. Again, there are two cases:

(2.1) RecSE fails. Clearly, in this case $\left(i_{k-1}+1, j_{k-1}+1\right)$ cannot be the source of $F_{k}$ which contradicts the assumption that $(i, j)$ is the source of $F_{1}$. We continue with the investigation of the next equality position from $L_{1}$.

(2.2) RecSE gives a (unique) solution, i.e., it is possible to reconstruct an $h v$ convex $S E$-directed polyomino $G$ with source $\left(i_{k-1}+1, j_{k-1}+1\right)$ and with the SCDR $\left\{i_{k-1}+1, \ldots, i^{\prime}\right\} \times\left\{j_{k-1}+1, \ldots, j^{\prime}\right\}$, where $i_{k-1}+1 \leq i^{\prime} \leq m$ and $j_{k-1}+1 \leq j^{\prime} \leq n$. Depending on the properties of $G$ we have two cases, again:

(2.2.1) If $\left(i^{\prime}, j^{\prime}\right) \neq(m, n)$ then $F_{k}$ cannot be the last component. Then, on the base of Theorem [2, $F_{k}$ is $N W$-directed and therefore $f_{i_{k}, j_{k}}=$ 1 (on the base of Lemma 1). If $g_{i^{\prime}, j^{\prime}} \neq 1$ then, clearly, $F_{k} \neq G$ which contradicts the assumption that $(i, j)$ is the source of $F_{1}$. We continue with the investigation of the next equality position from $L_{1}$. Otherwise, that is, when $g_{i^{\prime}, j^{\prime}}=1$, we can assume that $F_{k}=G$. On the base of Corollary 2 $G$ cannot be the first component of any other solution of the same type therefore $\left(i^{\prime}, j^{\prime}\right)$ can be deleted from $L_{1}$ and we continue with the next iteration.

(2.2.2) If $\left(i^{\prime}, j^{\prime}\right)=(m, n)$ then $F_{k}=G$ and $F=F_{1} \cup \ldots \cup F_{k}$. We found a solution and we continue with the investigation of the next equality position from $L_{1}$ in order to find another solutions. 

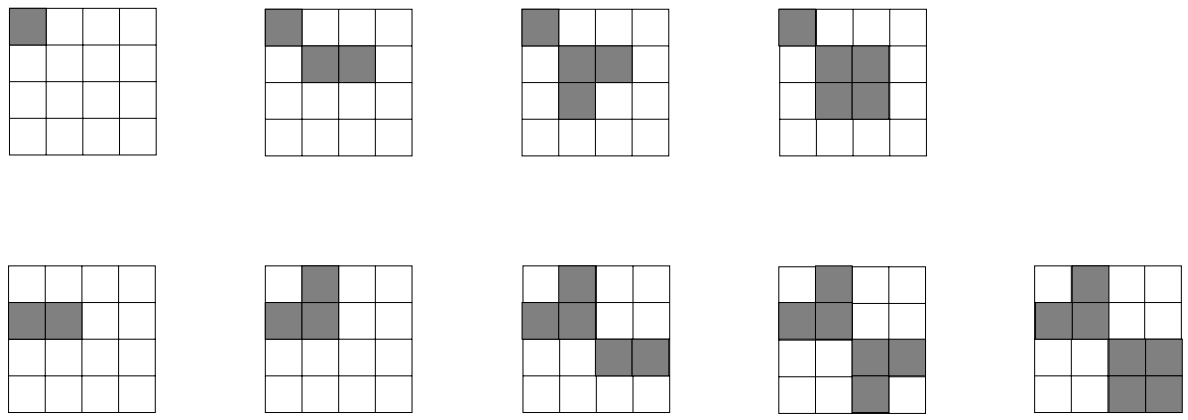

Fig. 4. Reconstructing sets of $\mathcal{S}_{8}^{\prime}$ of type 1 having projections $H=(1,2,2,2)$ and $V=(1,2,2,2) . L_{1}=\{(1,1),(2,2),(3,3)\}$. 1st row: trying to build a solution from position $(1,1)$ by filling a row or a column in each step the algorithm fails because of no place for filling the last row. After this step $L_{1}=\{(2,2)\}$ since position $(3,3)$ can be deleted from $L_{1}$ (see Case (2.2.1) of the algorithm). 2nd row: testing the only position $(2,2)$ from $L_{1}$ whether it is the source of $F_{1}$, the only solution of type 1 is found.
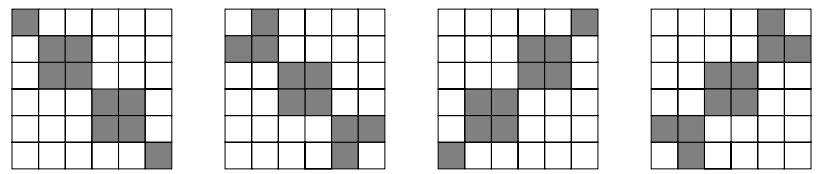

Fig. 5. Four $h v$-convex 8-connected but not 4-connected discrete sets with the same row and column sums: $H=(1,2,2,2,2,1), V=(1,2,2,2,2,1)$.

The first part of our algorithm (searching for solutions of type 1) is illustrated in Fig. 4 The second part of the algorithm, i.e., when it is assumed that $F$ has type 2 , is similar to the first part but we investigate equality positions of type 2 instead of type 1 and try to build $N E$ - and $S W$-directed components from the corresponding sources (using the algorithms RecNE and RecSW).

If no solutions are found after investigating all equality positions of both types then the assumption that $F \in \mathcal{S}_{8}^{\prime}$ is not met, i.e., there is no discrete set with the given projections which is $h v$-convex, 8-connected but not 4 -connected. However, in some cases there can be more than one solution (see Fig. [5).

Theorem 4. The worst case computational complexity of Algorithm REC8' is of $O(m n \cdot \min \{m, n\})$. The algorithm finds all sets of $\mathcal{S}_{8}^{\prime}$ with the given projections.

Proof. Every row and column index can be in an equality position of both type at most once. This means that we have at $\operatorname{most} \min \{m, n\}$ equality positions of type 1 and at most $\min \{m, n\}$ equality positions of type 2. Moreover, equality positions can be found in time $O(m+n)$ by Algorithms L_1 and L_2. Building the $\mathcal{S}_{4}$-components of $F$ assuming that an equality position $(i, j)$ is in $C_{F}$ takes $O(m n)$ time. We have to examine every equality position if it is in $C_{F}$, so we get the execution time $O(m n \cdot \min \{m, n\})$ in the worst case. 
Table 1. Average execution times in seconds of Algorithm REC8' and Algorithm C depending on the size of the matrix. Each set of test data consists of $1000 h v$-convex 8-connected but not 4-connected discrete sets.

\begin{tabular}{|c|c|c|}
\hline Size $n \times n$ & Algorithm REC8 & Algorithm C \\
\hline \hline $20 \times 20$ & 0.000272 & 0.011511 \\
\hline $40 \times 40$ & 0.001064 & 0.032524 \\
\hline $60 \times 60$ & 0.002597 & 0.065897 \\
\hline $80 \times 80$ & 0.004746 & 0.116505 \\
\hline $100 \times 100$ & 0.007831 & 0.178633 \\
\hline
\end{tabular}

On the base of Theorems 2 and 3 the sets reconstructed by Algorithm REC8' are $h v$-convex, 8-connected and have the given projections $H$ and $V$.

On the base of Theorem 3 any element of $C_{F}$ together with the projections and the knowledge of the type of $F$ is sufficient to reconstruct $F$ uniquely. Elements of $C_{F}$ are equality positions, too, on the base of Lemma 2, Since Algorithm REC8' examines every equality position whether it is in $C_{F}$, the second part of the theorem follows.

\subsection{Experimental Results}

In 2001 E. Balogh et al. presented an algorithm having worst case complexity of $O\left(m n \cdot \min \left\{m^{2}, n^{2}\right\}\right)$, which has so far the best average time complexity for reconstructing $h v$-convex 8-connected discrete sets (Algorithm $\mathrm{C}$ in 2]). In order to compare the average execution times of our Algorithm REC8' and Algorithm $\mathrm{C}$ we need to generate sets of $\mathcal{S}_{8}^{\prime}$ at random with uniform distribution. In [2] an algorithm is also given to generate $h v$-convex 8 -connected discrete sets having fixed row and column numbers with uniform distribution. The method is also suitable to generate sets od $\mathcal{S}_{8}^{\prime}$ with uniform distribution (we check whether the generated set is 4 -connected and if so, then we simply omit it).

We have generated discrete sets of $\mathcal{S}_{8}^{\prime}$ with different sizes. Then, we have reconstructed them with both algorithms. We used a PC with AMD Athlon processor of $1.4 \mathrm{GHz}$ and 1.5 GB RAM under Red Hat Linux release 7.3. The programs were written in $\mathrm{C}++$. The average execution times in seconds for obtaining all the solutions of different test sets are presented in Table 1 The results show that not only the worst case complexity of our algorithm is better (see Theorem 4) but also its average execution time was much better using any of the five test sets.

\section{Conclusions}

We have introduced a subclass of $h v$-convex 8-connected discrete sets, the class of $h v$-convex 8- but not 4-connected sets, and investigated the reconstruction problem in this class. We have shown that sets belonging to this class can be decomposed into so-called $\mathcal{S}_{4}$-components which can be uniquely reconstructed. We also introduced the concept of equality positions in order to determine these components. 
A reconstruction algorithm has been given with worst case complexity of $O(m n \cdot \min \{m, n\})$ and compared to a previous (more general) one given in [2]. It is quite surprising that the assumption on a set being 8-connected but not 4-connected makes so much improving in the reconstruction complexity (c.f. [13]).

These results give us a better understanding of the reconstruction problems and hopefully lead us towards designing reconstruction algorithms in broader classes of discrete sets.

\section{References}

1. P. Balázs, E. Balogh, A. Kuba, Reconstruction of 8-connected but not 4-connected discrete sets, Technical Report at the University of Szeged (2002) http://www.inf.u-szeged.hu/〜pbalazs/research/research.html

2. E. Balogh, A. Kuba, Cs. Dévényi, A. Del Lungo, Comparison of algorithms for reconstructing $h v$-convex discrete sets, Lin. Alg. and Its Appl. 339 (2001) 23-35.

3. E. Barcucci, A. Del Lungo, M. Nivat, R. Pinzani, Reconstructing convex polyominoes from horizontal and vertical projections, Theor. Comput. Sci. 155 (1996) 321-347.

4. S. Brunetti, A. Daurat, Reconstruction of discrete sets from two or more X-rays in any direction, Proceedings of the seventh International Workshop on Combinatorial Image Analysis (2000) 241-258.

5. S. Brunetti, A. Del Lungo, F. Del Ristoro, A. Kuba, M. Nivat, Reconstruction of 8and 4-connected convex discrete sets from row and column projections, Lin. Alg. and Its Appl. 339 (2001) 37-57.

6. M. Chrobak, Ch. Dürr, Reconstructing $h v$-convex polyominoes from orthogonal projections, Information Processing Letters 69(6) (1999) 283-289.

7. A. Del Lungo, M. Nivat, R. Pinzani, The number of convex polyominoes reconstructible from their orthogonal projections, Discrete Math. 157 (1996) 65-78.

8. G.T. Herman, A. Kuba (Eds.), Discrete Tomography, Special Issue. Int. J. Imaging Systems and Techn. 9 (1998) No. 2/3.

9. G.T. Herman, A. Kuba (Eds.), Discrete Tomography: Foundations, Algorithms and Applications (Birkhäuser, Boston, 1999).

10. A. Kuba, The reconstruction of two-directionally connected binary patterns from their two orthogonal projections, Comp. Vision, Graphics, and Image Proc. $\mathbf{2 7}$ (1984) 249-265.

11. A. Kuba, Reconstruction in different classes of 2D discrete sets, Lecture Notes on Computer Sciences 1568 (1999) 153-163.

12. A. Kuba, E. Balogh, Reconstruction of convex $2 \mathrm{D}$ discrete sets in polynomial time, Theor. Comput. Sci. 283 (2002) 223-242.

13. L. Latecki, U. Eckhardt, A. Rosenfeld, Well-Composed Sets, Computer Vision and Image Understanding 61(1) (1995) 70-83.

14. H.J. Ryser, Combinatorial properties of matrices of zeros and ones, Canad. J. Math. 9 (1957) 371-377.

15. G.W. Woeginger, The reconstruction of polyominoes from their orthogonal projections, Information Processing Letters 77 (2001) 225-229. 